EPJ Web of Conferences 61, 04010 (2013)

DOI: $10.1051 /$ epjconf/ 20136104010

(C) Owned by the authors, published by EDP Sciences, 2013

\title{
An Exceptional Radio Flare in Markarian 421
}

\author{
Joseph L. Richards ${ }^{1, a}$, Talvikki Hovatta ${ }^{2}$, Matthew L. Lister ${ }^{1}$, Anthony C. S. Readhead ${ }^{2}$, Walter Max-Moerbeck ${ }^{3}$, \\ Tuomas Savolainen ${ }^{4}$, Emmanouil Angelakis ${ }^{4}$, Lars Fuhrmann ${ }^{4}$, Margo F. Aller ${ }^{5}$, Hugh D. Aller ${ }^{5}$, Ioannis Myserlis ${ }^{4}$, \\ and Vassilis Karamanavis ${ }^{4}$ \\ ${ }^{1}$ Purdue University \\ ${ }^{2}$ California Institute of Technology \\ ${ }^{3}$ NRAO \\ ${ }^{4}$ MPIfR \\ ${ }^{5}$ University of Michigan
}

\begin{abstract}
In September 2012, the high-synchrotron-peaked (HSP) blazar Markarian 421 underwent a rapid wideband radio flare, reaching nearly twice the brightest level observed in the centimeter band in over three decades of monitoring. In response to this event we carried out a five epoch centimeter- to millimeter-band multifrequency Very Long Baseline Array (VLBA) campaign to investigate the aftermath of this emission event. Rapid radio variations are unprecedented in this object and are surprising in an HSP BL Lac object. In this flare, the $15 \mathrm{GHz}$ flux density increased with an exponential doubling time of about 9 days, then faded to its prior level at a similar rate. This is comparable with the fastest large-amplitude centimeter-band radio variability observed in any blazar. Similar flux density increases were detected up to millimeter bands. This radio flare followed about two months after a similarly unprecedented $\mathrm{GeV}$ gamma-ray flare (reaching a daily $E>100 \mathrm{MeV}$ flux of $\left.(1.2 \pm 0.7) \times 10^{-6} \mathrm{ph} \mathrm{cm}^{-2} \mathrm{~s}^{-1}\right)$ reported by the Fermi Large Area Telescope (LAT) collaboration, with a simultaneous tentative TeV detection by ARGO-YBJ. A cross-correlation analysis of long-term $15 \mathrm{GHz}$ and LAT gamma-ray light curves finds a statistically significant correlation with the radio lagging $\sim 40$ days behind, suggesting that the gamma-ray emission originates upstream of the radio emission. Preliminary results from our VLBA observations show brightening in the unresolved core region and no evidence for apparent superluminal motions or substantial flux variations downstream.
\end{abstract}

\section{Introduction}

Markarian 421 (Mrk 421, $z=0.031$ ) is one of the nearest, best known, and most intensively studied blazars (including four talks and two posters at this meeting, [1-5]). It is classified as a BL Lac object and was the first extragalactic gamma-ray source detected at $\mathrm{TeV}$ energies [6]. At these energies it is one of the brightest and fastest-varying extragalactic objects, with a history of strong variations on timescales of hours or less [7]. It is also a bright X-ray source, and is frequently detected in GeV gamma-rays.

Based on its spectral energy distribution (SED), which shows a synchrotron peak in the X-ray band, Mrk 421 is classified as a high-synchrotron-peaked (HSP) object [8]. Its radio behavior is rather tame, typical of HSP blazars [e.g., 9, 10]. As illustrated in the left panel of figure 1 , no major variability was detected in more than 30 years of regular monitoring at $14.5 \mathrm{GHz}$ by the University of Michigan Radio Astronomical Observatory (UMRAO). Extensive studies using very long baseline interferometry (VLBI) have not found evidence for apparent superluminal motion in the components in the parsec-scale regions around the core [e.g., 11-13].

\footnotetext{
a e-mail: jlr@purdue.edu
}

In September 2012, an unprecedented radio flare was detected by the Owens Valley Radio Observatory (OVRO) $40 \mathrm{~m}$ blazar monitoring program [14, 15]. The OVRO $15 \mathrm{GHz}$ flux densities are plotted in green in the right panel of figure 1. In response to this unexpected radio event, we executed a rapid-cadence five-epoch polarimetric monitoring campaign with the Very Long Baseline Array (VLBA) to investigate whether any parsec-scale structural or kinematic changes occurred. We present here preliminary results from our U-band total intensity VLBA observations and from our single-dish monitoring.

\section{Details of the Flare}

The first signs of the radio flare that peaked in September 2012 appeared in mid-August 2012. In late August and September, the OVRO $15 \mathrm{GHz}$ flux density measurements of Mrk 421 increased exponentially from about $550 \mathrm{mJy}$ to a peak of $(1.09 \pm 0.03) \mathrm{Jy}$ on 21 September 2012 with a doubling time of about 9 days. As can be seen in figure 1 , the flux density then returned to a lower but still elevated level along a similar exponential profile. This is extremely rapid radio variation for a blazar. A study of about 500 flares in about 80 blazars at 22 and $37 \mathrm{GHz}$, 

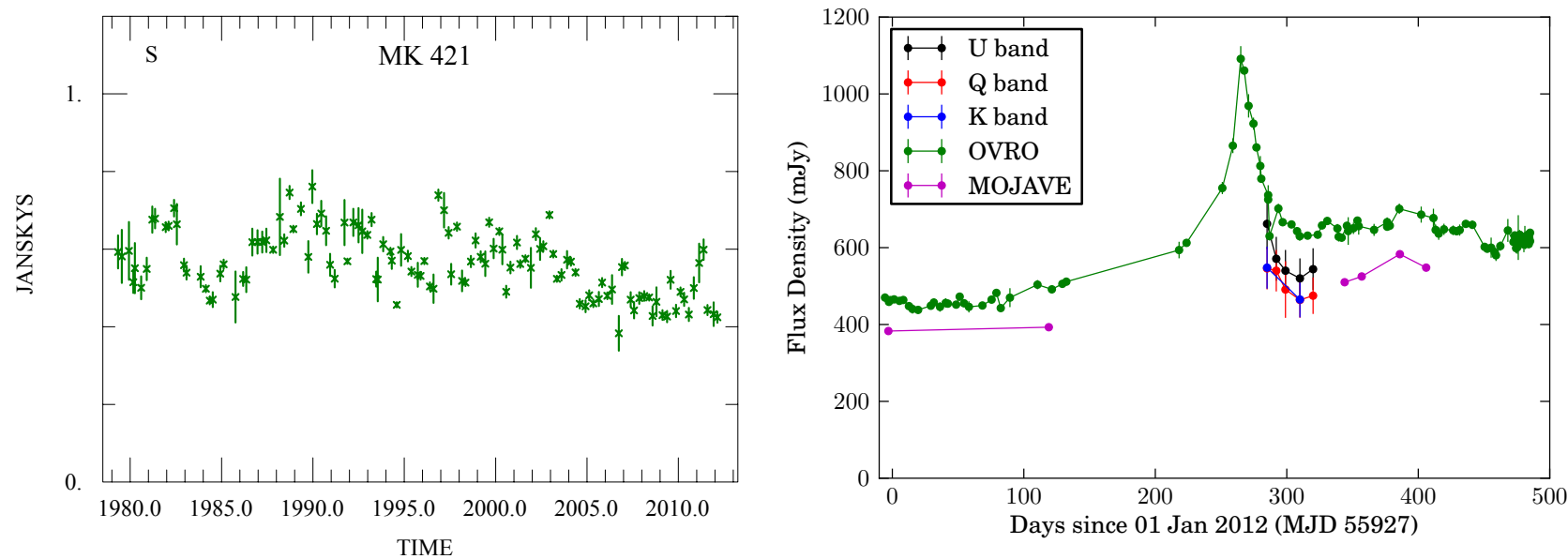

Figure 1. Long-term radio light curves for Mrk 421, including UMRAO $14.5 \mathrm{GHz}$ data (left panel) and OVRO $15 \mathrm{GHz}$, MOJAVE $15 \mathrm{GHz}$, and our multifrequency VLBA data (right panel). Vertical scales are equal in both panels.

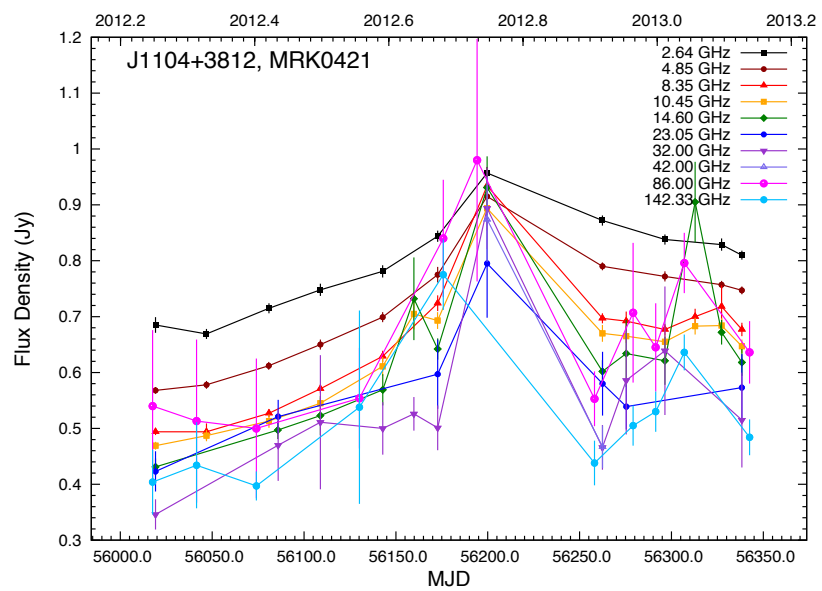

Figure 2. F-GAMMA radio light curves from 2.6 to $142 \mathrm{GHz}$.

where variability is typically faster and stronger than at $15 \mathrm{GHz}$, found a median doubling time of about 60 days and none faster than 9 days [16].

The flare at $15 \mathrm{GHz}$ was accompanied by nearly simultaneous flaring at all observed frequencies from 2.6 to $142 \mathrm{GHz}$. These multiband radio observations, shown in figure 2, were obtained through the F-GAMMA program ${ }^{1}$ as part of an ongoing gamma-ray blazar monitoring campaign. The F-GAMMA light curves appear to show a doubly-peaked flare profile at several frequencies including $14.6 \mathrm{GHz}$, with an earlier peak near 16 August 2012, but the uncertainty on these points is high. OVRO data about a week earlier do not show signs of this early peak.

This multiband radio flare followed shortly after a major GeV gamma-ray outburst reported by the Fermi Large Area Telescope (LAT) Collaboration [17]. A tentative detection at $\mathrm{TeV}$ energies coincident with the $\mathrm{GeV}$ flare was also reported [18], but other multiwavelength follow-up during and after this flare was precluded because Mrk 421 was too near the Sun. Later multiwavelength observations

${ }^{1}$ http://www3.mpifr-bonn.mpg.de/div/vlbi/fgamma/fgamma.html of the source in early 2013 found major variability and flaring in many bands [e.g., 19, and related ATels].

We can estimate the Doppler factor of the jet from the radio variability, following [16]. Assuming the light travel time across the emission region sets the variability timescale, we can estimate the brightness temperature of the emission region during the flare to be

$$
T_{\text {b,var }}=\left(1.548 \times 10^{-32} \mathrm{~K}\right) \times \frac{\Delta S d_{\mathrm{L}}^{2}}{v^{2} \tau^{2}(1+z)},
$$

where $\Delta S \approx 0.5 \mathrm{Jy}$ is the amplitude of the flare in janskies, $d_{\mathrm{L}}=133 \mathrm{Mpc}=4.1 \times 10^{24} \mathrm{~m}$ is the luminosity distance to Mrk 421 in meters, $v=15 \mathrm{GHz}$ is the observation frequency in $\mathrm{GHz}, \tau \approx 13$ days is the exponential time constant for the flare in days, and $z=0.031$ is the redshift. With these parameters, we find $T_{\mathrm{b} \text {,var }} \approx 3.3 \times 10^{12} \mathrm{~K}$. Assuming the rest-frame brightness temperature of the emission region is near the equipartition limit, $T_{\mathrm{b} \text {,eq }} \approx 5 \times 10^{10} \mathrm{~K}$ [20], we estimate the Doppler factor to be

$$
D_{\mathrm{var}}=\left(\frac{T_{\mathrm{b}, \mathrm{var}}}{T_{\mathrm{b}, \mathrm{eq}}}\right)^{1 / 3} \approx 4.1 .
$$

This modest value is in accord with previous estimates of the Doppler and Lorentz factors based on VLBI data [e.g., $11,13]$. Estimates of the Doppler factor from high energy observations find much higher values, $D \sim 20$ [e.g., 21]. This apparent conflict thus remains, perhaps indicating a velocity structure in the jet [see $1,22,23$, for related discussions in these proceedings].

\section{VLBA Follow-Up Observations}

In response to the September 2012 extreme radio flare, we undertook a multiwavelength polarimetric VLBA monitoring campaign to characterize the structure and kinematics of the parsec-scale jet. The total flux density at $15 \mathrm{GHz}$ ( $\mathrm{U}$ band), $24 \mathrm{GHz}$ (K band), and $43 \mathrm{GHz}$ (Q band) are plotted in figure 1 . The first epoch was observed on 12 October, 2012, about 21 days after the peak of the $15 \mathrm{GHz}$ 


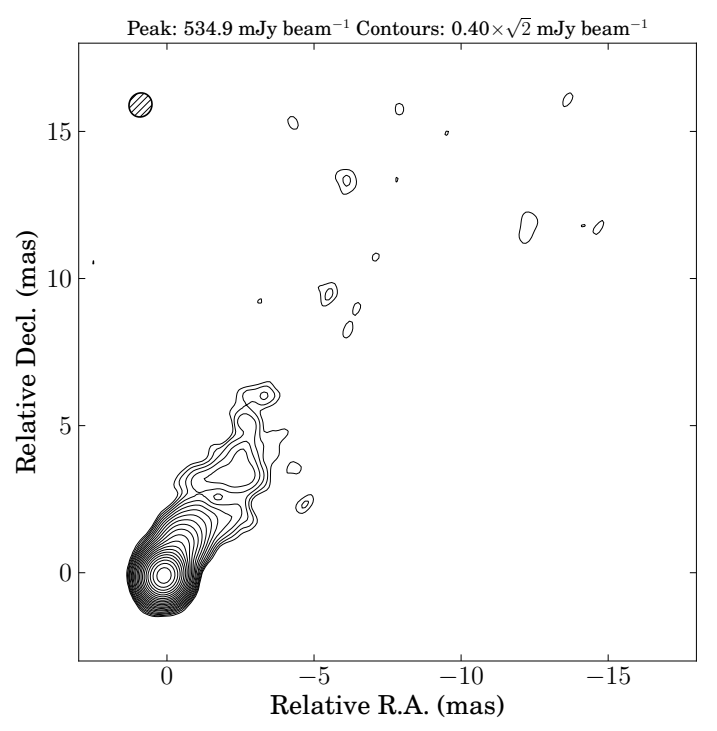

Figure 3. Total intensity contour map from the 12 October 2012 U-band $(15 \mathrm{GHz})$ VLBA observation.

Table 1. Apparent component speeds

\begin{tabular}{cc}
\hline Component & Apparent Speed \\
\hline 1 & $(0.3 \pm 1.8) c$ \\
2 & $(3.8 \pm 2.1) c$ \\
3 & $(1.9 \pm 0.9) c$ \\
4 & $(1.2 \pm 0.4) c$ \\
5 & $(0.2 \pm 0.1) c$ \\
\hline
\end{tabular}

flare. At this time, the flux density had dropped substantially from its peak.

We reduced our VLBA data using standard methods with AIPS and Difmap [24]. A total intensity contour map for the U-band data from our first VLBA observation is shown in figure 3 . This map differs little morphologically from the MOJAVE ${ }^{2}$ U-band map obtained about five months earlier on 29 April 2012. Figure 4 shows the difference between these maps, which is largely confined to the core region. Our K- and Q-band observations show a very similar morphology. Preliminary analysis of our subsequent U-band total intensity observations show a fading of the core region, but no major structural changes.

To examine the kinematics of the jet, we used Difmap to fit a model consisting of an elliptical Gaussian or deltafunction core (depending on epoch) and circular Gaussian downstream components to our $15 \mathrm{GHz}(u, v)$ data. The model for the first epoch is shown in figure 5. We obtained reasonable reduced $\chi^{2}$ in all epochs using a six-component model. The radial distance from each downstream component to the core component in all five epochs is plotted in figure 6. By fitting a linear trend for each component, we determined their apparent speeds, tabulated in table 1. All are consistent (to $2 \sigma$ ) with stationarity or subluminal speed.

\footnotetext{
${ }^{2}$ http://www.physics.purdue.edu/mojave
}

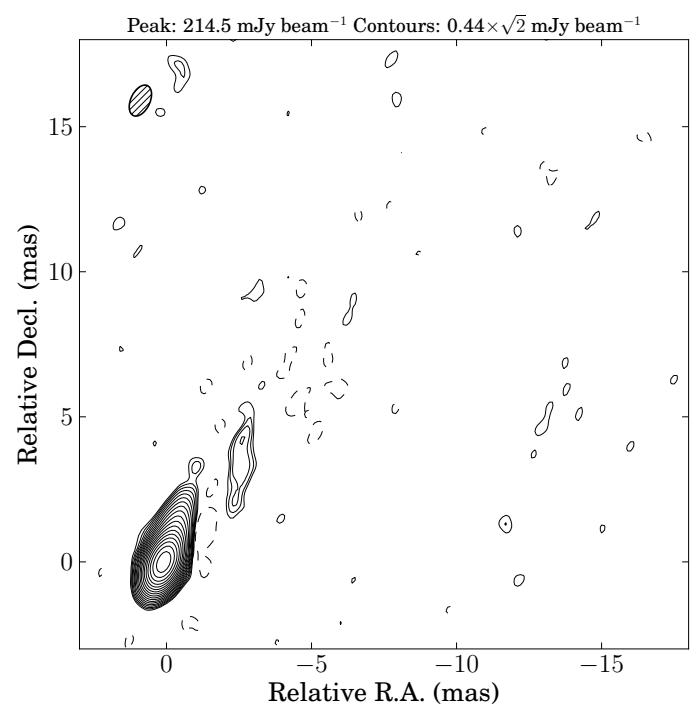

Figure 4. Difference in total intensity between the 12 October 2012 U-band map and the preceding MOJAVE epoch, observed 29 April 2012. Before subtracting, both cleaned maps were restored with the elliptical Gaussian beam whose position angle and FWHM size is shown in the upper left.

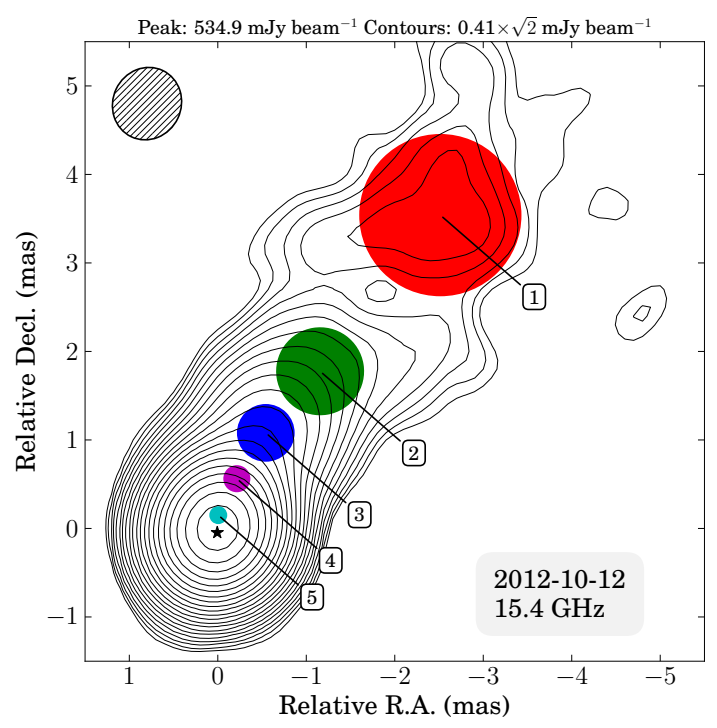

Figure 5. Fitted components overlaid on the 12 October 2012 Uband total intensity contour map. Components are circular Gaussians with FWHM illustrated by the size of the plotted circles. The core is modeled as a delta-function component, plotted as a star.

\section{Radio/Gamma-ray Cross Correlation}

One must exercise caution when concluding that a physical connection exists between individual flares based solely on coincidence in time. Because of the extremely rare nature of the radio flare and the infrequency of gamma-ray flares, this particular case is more convincing than many. Still, a quantitative estimate of the significance of the occurrence of two flares is desirable. To estimate significances, we have performed a set of Monte Carlo simulations. 


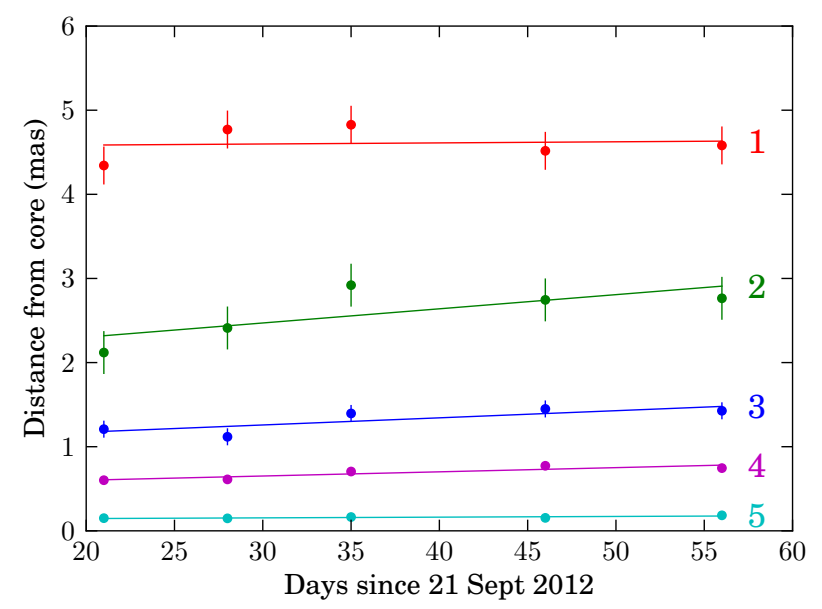

Figure 6. Radial distance of each component in the U-band model fit at each epoch.

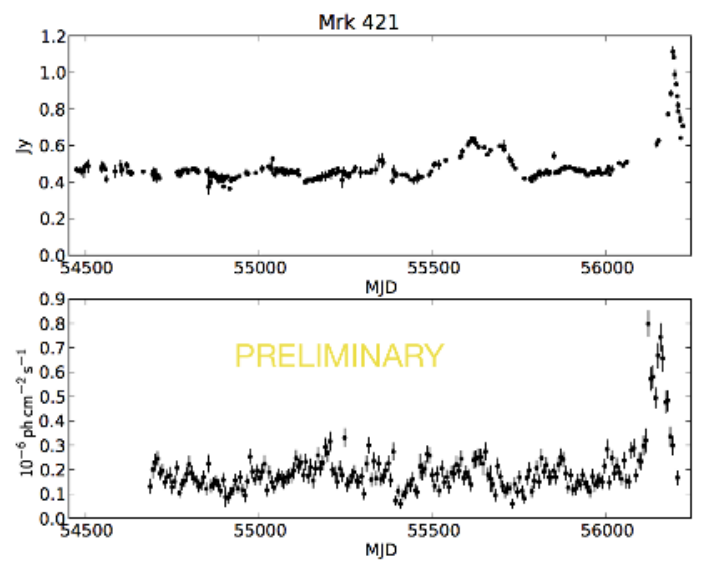

Figure 7. OVRO $40 \mathrm{~m} 15 \mathrm{GHz}$ radio light curve (top) and FermiLAT $100 \mathrm{MeV}-200 \mathrm{GeV}$ weekly binned gamma-ray light curve for Mrk 421.

In figure 7, the preliminary weekly-binned LAT light curve and the OVRO $15 \mathrm{GHz}$ light curves are shown. The discrete cross-correlation function (DCF) of these two light curves, computed following [25] to account for uneven sampling, is shown in figure 8 . The positive and negative contours show the $1 \sigma, 2 \sigma$, and $3 \sigma$ significance levels. These are determined by simulating 20,000 mock radio and gamma-ray light curves using the same uneven sampling as the original data sets. The light curves are assumed to result from red noise processes with noise power spectral densities proportional to $f^{-\beta}$, using the true light curves to estimate $\beta$ separately for each band. Further details of this method and a previous discussion of this Mrk 421 event are found in [26].

The DCF peak we find is nearly at the $3 \sigma$ significance level. Very few blazars in the OVRO monitoring program reach this level of significance according to our method. If we exclude the flaring period, the significance of the highest peak drops below $1 \sigma$. Thus, our method agrees with

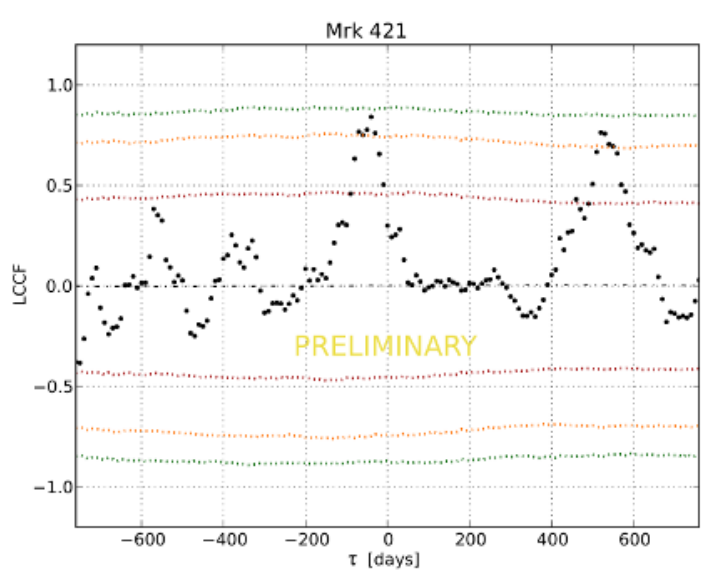

Figure 8. Discrete cross-correlation function between the radio and gamma-ray light curves shown in figure 7. Dashed lines indicate the positive and negative $1 \sigma, 2 \sigma$, and $3 \sigma$ significance contours obtained through Monte Carlo simulation.

our intuitive sense that these light curves show a true correlation. However, there is ambiguity in this result: there are two peaks in the DCF, both of similar amplitude and significance. The peak at -40 days results from the overlap of the September radio flare with the gamma-ray flare. The second peak, near a lag of 500 days, results from the overlap of the smaller radio high state apparent near MJD 55650 in the radio light curve in figure 7. Either pairing of events is unlikely to occur by chance, but with only a single major feature in the gamma-ray light curve we cannot unambiguously determine the lag from the light curves alone. Because of their similar appearances in the plot, it is tempting to conclude that the larger radio flare corresponds to the gamma-ray flare, but this implicitly assumes a model for the physical process connecting the emission in the two bands.

It is important to note that for this test we explicitly selected a time interval including the flaring period in both bands. As such, our statistical result is a posteriori, and does not represent independent statistical confirmation of this correlation. To claim such a confirmation, we would require the comparison of long light curves with a time interval selected independently of source activity. For now, the chief evidence supporting this is the temporal coincidence of rare events.

\section{Conclusions}

Mrk 421 displayed a radio emission event unique in its long radio monitoring history. The small delay between this extremely rare event and a preceding $\mathrm{GeV}$ and possibly $\mathrm{TeV}$ gamma-ray flare, both infrequent occurrences in this source, provides intuitively compelling evidence for a physical connection between them. Our quantitative method for finding significant correlations between light curves supports this conclusion, although it does not provide independent evidence. If the gamma-ray flare and the September 2012 radio flare are, in fact, related, there is a 
delay of about 40 days from the gamma-ray flare to the radio flare. Preliminary analysis of the $15 \mathrm{GHz}$ radio light curve and our VLBA follow-up observations does not find evidence for the ejection of a superluminal component. Our data are consistent with previous radio determinations of the jet Doppler factor, $D \approx 3$. Thus, there remains a conflict between this and the much higher Doppler factors required by the high-energy emission. It seems that if this is indicative of velocity structure, even in this unusual radio flare, we did not detect radio emission from a putative faster region of the jet.

\section{Acknowledgements}

J. R. and T. H. acknowledge the support of the American Astronomical Society and the National Science Foundation (NSF) in the form of International Travel Grants. T. H. was supported by the Jenny \& Antti Wihuri foundation. This work and the MOJAVE program were supported by NASA Fermi Guest Investigator grant 11-Fermi11-0019. The OVRO $40 \mathrm{~m}$ monitoring program is supported in part by NSF grants AST0808050 and AST-1109911 and NASA grants NNX08AW31G and NNX11AO43G. UMRAO was supported in part by NSF grant AST-0607523 and NASA Fermi Guest Investigator grants NNX09AU16G, NNX10AP16G, and NNX11AO13G. The National Radio Astronomy Observatory is a facility of the National Science Foundation operated under cooperative agreement by Associated Universities, Inc.

\section{References}

[1] R.L. Lico et al., these proceedings (2013)

[2] K. Niinuma et al., these proceedings (2013)

[3] A. Mastichiadis, M. Petropoulou, S. Dimitrakoudis, these proceedings (2013)

[4] I.M. Balokovic et al., these proceedings (2013)

[5] E. Racero, I. de la Calle, these proceedings (2013)
[6] M. Punch et al., Nature 358, 477 (1992)

[7] J.A. Gaidos et al., Nature 383, 319 (1996)

[8] A.A. Abdo et al., ApJ 736, 131 (2011)

[9] B.G. Piner, N. Pant, P.G. Edwards, ApJ 723, 1150 (2010)

[10] M. Ackermann et al., ApJ 743, 171 (2011)

[11] B.G. Piner, P.G. Edwards, ApJ 622, 168 (2005)

[12] M.L. Lister et al., AJ 138, 1874 (2009)

[13] R. Lico et al., A\&A 545, A117 (2012)

[14] J.L. Richards et al., ApJS 194, 29 (2011)

[15] T. Hovatta et al., The Astronomer's Telegram \#4451 (2012)

[16] T. Hovatta et al., A\&A 494, 527 (2009)

[17] F. D'Ammando et al., The Astronomer's Telegram \#4261 (2012)

[18] B. Bartoli et al., The Astronomer's Telegram \#4272 (2012)

[19] M. Balokovic et al., The Astronomer's Telegram \#4974 (2013)

[20] A.C.S. Readhead, ApJ 426, 51 (1994)

[21] L. Maraschi et al., ApJ 526, L81 (1999)

[22] G. Piner, P.G. Edwards, these proceedings (2013)

[23] M.L. Lister et al., these proceedings (2013)

[24] M.C. Shepherd, T.J. Pearson, G.B. Taylor, DIFMAP: an interactive program for synthesis imaging., in Bulletin of the American Astronomical Society (1994), Vol. 26, pp. 987-989

[25] R.A. Edelson, J.H. Krolik, ApJ 333, 646 (1988)

[26] W. Max-Moerbeck et al., OVRO $40 \mathrm{~m}$ Blazar Monitoring Program: Location of the gamma-ray emission region in blazars by the study of correlated variability at radio and gamma-rays, in Fourth International Fermi Symposium Proceedings, edited by T.J. Brandt, N. Omodei, C. Wilson-Hodge (eConf C121028, 2013), p. 252, [astro-ph:1303.2131] 